\title{
One or two different sets of laws of learning- Is this an empirical question?
}

\author{
SAM S. RAKOVER \\ Oakland University, Rochester, Michigan 48063
}

\begin{abstract}
Theoretical arguments were developed to show that the answer to the question posed in the title is in the negative. First, it has been shown that one cannot answer the question by making empirical observations. Second, the answer, a unified theory of learning, is directly derived from the self-evident propositions of science.
\end{abstract}

\section{CLARIFICATIONS}

\section{Some Clarifications of the Question}

One of the most enduring problems to be found in the psychology of learning concerns the theoretical distinction between classical and instrumental conditioning. The first suggestion that these two experimental procedures manifest two different learning processes was made by S. Miller and Konorski (1928) and was further developed by others (e.g., Kimble, 1961). The two-factor theory contrasts the unifactor theory, according to which both procedures demonstrate one set of laws of learning. These distinctions have been expressed in different ways, but as Mackintosh (1974, p. 125) wrote, "essentially, however, these must reduce to the question whether the operational distinction between classical conditioning and instrumental learning implies that different learning processes are engaged in the two situations."

No serious systematic attempt was made to resolve this problem empirically until about 1965 . Since then, the "one or two different laws of learning" problem has been systematically attacked in an attempt to support the unifactor theory. It has been argued that if the laws of classical conditioning are the same as the laws of instrumental learning, then every response that has been conditioned classically can be learned instrumentally (e.g., Mackintosh, 1974; N. E. Miller, 1969).

Since the arguments to follow will not concern incompatible empirical results, problems of replication, or any other methodological pitfalls, an ideal condition in which all these problems have been satisfactorily resolved shall be assumed (e.g., N. E. Miller \& Dworkin, 1974). A "bipotential learning phenomenon" is proposed, according to which classically conditioned responses $(\mathrm{Rc})$ can be learned instrumentally and

The writing of this article was funded by Oakland University. The author wishes to thank Clark Heston, Ted Landau, Keith Stanovich, David Ronis, David Stonner, and Harvey Burdick, who read an earlier draft of this manuscript and offered helpful suggestions. Requests for reprints should be addressed to Sam S. Rakover, Department of Psychology, Oakland University, Rochester, Michigan 48063. instrumentally learned responses $(\mathrm{Ri})$ can be conditioned classically. This is a general assumption that includes both lines of empirical effort to show a unified theory of learning: (1) that $\mathrm{Ri}$ and any other learnable response, such as pecking in pigeons, can be conditioned classically; and (2) that Rc and any other learnable response can be learned instrumentally.

A second assumption is proposed, according to which there is one theory and one set of laws or learning processes $\left(\mathrm{L}_{1}\right)$ that is associated with classical conditioning procedure, and another set $\left(\mathrm{L}_{2}\right)$ that is associated with instrumental learning procedure. The latter assumption takes into account the various differences that have been suggested to exist between classical and instrumental learning, such as the type of neurological mechanisms (e.g., autonomic vs. central nervous system) and the type of responses (e.g., elicited vs. emitted). It should be noted that the above two assumptions represent a special type of relationship among two different sets of laws of learning, two different methods of teaching, and two different kinds of responses.

\section{Some Clarifications of the Meaning \\ of "Empirical Question"}

Without going into a debate concerning the criticisms on the distinction between the so-called theoretical and observational languages and the line of demarcation between science and pseudoscience (e.g., Suppe, 1974), for the sake of the present paper, it will be sufficient to describe these concepts operationally. Empirical observations will be described in terms of the results of experiments, theories in terms of the apparatus generating predictions whether these observations will occur or not, and a nonempirical question will be referred to as one for which an answer can be given without having made empirical observations. The latter is a statement for which the truth value can be determined from a set of given self-evident concepts and postulates. For example, the statement, "John's behavior is caused," is not an empirical one because this statement is no more than a paraphrasing of the implicit or explicit basic assumption of science, that 
nature is ordered. A scientist usually does not deal with questions of this kind. Rather, he searches for specific causes and mechanisms by which John's behavior can be explained. It will be argued that the "one or two different laws of learning" problem is of the same type as the question, "Is John's behavior caused?" The answer to both questions is derived directly from the self-evident propositions of science.

\section{ARGUMENTS}

\section{Arguments Demonstrating that One Cannot \\ Show that $L_{1}$ is the Same as $L_{2}$ by \\ Making Empirical Observations}

Given that $\mathrm{L}_{1}$ and classical conditioning procedure explain $\mathrm{Rc}$, and that $\mathrm{L}_{2}$ and instrumental learning procedure explain $\mathrm{Ri}$ and the "bipotential learning phenomenon," it is not necessarily true that $L_{1}$ and $L_{2}$ are the same set of laws. The reason for this is simply that it is possible to offer many different explanations for any empirical demonstration. $\mathrm{L}_{1}$ and $\mathrm{L}_{2}$ are only two possible explanations for the "bipotential learning phenomenon." If we conclude that $\mathrm{L}_{1}$ and $\mathrm{L}_{2}$ are the same set of laws since they entail the same result, then by the same logic we should also conclude that $\mathrm{y}_{1}=5 \mathrm{x}_{1}$ is the same function as $\mathrm{y}_{2}=3+2 \mathrm{x}_{2}^{2}$, because $\mathrm{y}_{1}=\mathrm{y}_{2}=5$ when $\mathrm{x}_{1}=\mathrm{x}_{2}=1$.

A further elaboration of this argument is as follows. $\mathrm{L}_{1}$ and $\mathrm{L}_{2}$ can be considered the same set of laws only if $\mathrm{L}_{1}$ is logically equated with classical conditioning procedure, which in turn is logically equated with $\mathrm{Rc}$, and $\mathrm{L}_{2}$ is equated with instrumental learning, which in turn is equated with $\mathrm{Ri}$. However, these requirements cannot be fulfilled. Although it is possible to conceive of learning as a disposition of an organism that is manifested in certain conditions of teaching, it is unjustified to equate these procedures with learnable behavior. The method of teaching does not entail learning in the same way dispositional terms are defined. For example, in physics, something is elastic only if after it is stretched and released, it contracts. In the latter case, it is possible to suggest that a particular disposition in something may be equated with the underlying state, which is causally responsible for the manifestation of this disposition. However, in the former case, except for the underlying state (i.e., the method of teaching), one needs to take into account the organism itself in order to explain learnable behavior (for a more complete discussion on a similar issue, see Cummins, 1974).

A further consideration in this argument is that it is unjustified to equate $L_{1}$ with classical and $L_{2}$ with instrumental procedures because they are only partial interpretations of $L_{1}$ and $L_{2}$. Both demonstrate the concept of learning by certain specific experimental procedures. Although classical and instrumental procedures may be conceived of as dispositional states by which learning is demonstrated, learning is not a "brute" disposition, such as the concepts of mass or gravitation in physics, for which as yet there exists no explanation (see Cummins, 1974). The main reason for this statement is that learning is explained by referring to a theory that shows how a certain response is learned by a certain method of teaching. Thus, since classical and instrumental learning are only ways or procedures for demonstrating $L_{1}$ and $L_{2}$, there exists the possibility that other different procedures might also manifest, although a bit differently, the same laws of learning. Therefore, there is no justification for equating $L_{1}$ with classical conditioning and $\mathrm{L}_{2}$ with instrumental conditioning. Thus, one can conclude that the empirical approach cannot provide us with an answer to this paper's question.

It is important to notice that the above analysis goes against the basic argument of the unifactor theory, which states that given $\mathrm{L}_{2}$ as a unified theory of learning, it is a logical necessity that Rc be learnable instrumentally. The reason for rejecting this deduction is that, since classical and instrumental learning are different partial interpretations of the theory of learning, it is possible that responses will be learned by one method but not by the other.

\section{Arguments Demonstrating that One Cannot \\ Show that $L_{1}$ is Different from $L_{2}$ by \\ Making Empirical Observations}

In view of the conclusions stated earlier, one might take the dualist approach (i.e., two-factor theory) and ask the following question: If it can be empirically shown that Rc cannot be learned instrumentally and that $\mathrm{Ri}$ cannot be conditioned classically, can it then be concluded that $L_{1}$ is qualitatively different from $L_{2}$ ? The answer is negative. Since classical and instrumental learning are nothing more than certain different teaching procedures, it is possible to teach certain behaviors with one procedure, but not with the other, without violating the possibility that both procedures are demonstrating the same theory. An analogy taken from the natural sciences will clarify this issue. It is predicted that a certain chemical reaction should result in the production of heat and radioactive radiation. Now, it is clear that the usual procedure of measuring temperature (e.g., utilizing a thermometer) cannot measure radiation, and the procedure of measuring radioactivity (e.g., utilizing a geiger counter) is not an appropriate procedure to measure temperature. Nevertheless, both procedures of measurement demonstrate the same theory. Therefore, although there exist many different ways of creating or producing different phenomena, with each phenomenon created by its own particular procedure, all are explained within the same unified theory. In the same manner, in the area of learning, there are different methods of teaching different behaviors; some responses can be taught by one method 
and not by another, but still, the explanation is to be derived from one theory.

\section{Arguments Demonstrating that the Answer to \\ This Paper's Question, a Unified Theory of Learning, is Directly Derived from the \\ Self-Evident Propositions of Science}

The discussion began with the question, "What is meant by two different laws of learning?" There are two ways by which one can state that there is a difference between $L_{1}$ and $L_{2}$ : (1) The predictions (P) derived from $L_{1}$ contradict the predictions derived from $L_{2}$, namely, $P$ is implied by $L_{1}$, while not-P is implied by $\mathrm{L}_{2}$; or (2) the basic concepts, the undefinable terms agreed upon, and the basic postulates of $\mathrm{L}_{1}$ and $\mathrm{L}_{2}$ are related to different contents or domains of learnable behavior. The first alternative was probably not assumed by either the two-factor or the unifactor theory. The main reason is that, according to the first alternative, there is only one set of laws of learning. The other set of laws should result in nonlearnable behavior. No one would say that the instrumental procedure produces learning, while Pavlovian procedure does not, or vice versa. Thus, what both theories imply is that these procedures demonstrate two different phenomena of learning.

The point has now been reached where the discussion concerns the self-evident propositions of science. The question is how one should proceed after specifying $L_{1}$ and $L_{2}$, and after showing that they do not contradict each other. The basic spirit of science is to develop a theory that explains $L_{1}$ and $L_{2}$, since the very essence of science is the search for a unified explanation of behavior (one way to achieve this goal is by theoretical reduction; see Sklar, 1967). This has been emphasized by many scientists and philosophers. Bronowski (1965), for example, expressed the idea very clearly: "Science is nothing else than the search to discover unity in the wild variety of nature-or more exactly, in the variety of our experience" (p. 16). It would violate the spirit of science to propose that $\mathrm{L}_{1}$ and $\mathrm{L}_{2}$ are two qualitatively different sets of laws of learning. Except that such a suggestion would lead to ad hoc explanations and nonparsimoniousness, it would undermine the development of science. In its extremity, this procedure will lead toward infinitely many theories of learning, that is, toward associating each experimental result with a different law of learning. Both Bronowski (1965) and Kuhn (1970) describe the main feature of scientific development as the emergence of one unified theory out of multitheories and explanations.

The implication of the "unity in variety" assumption of science for the present problem can also be demonstrated in another way. Empirical observations can answer questions of only one type: "Does a certain predicted phenomenon or property occur?" This fact has been utilized by scientists in order to confirm or refute various theories, that is, by comparing predictions with observations. Now, if we postulate one theory according to which $L_{1}$ and $L_{2}$ are the same set of laws, and a second theory according to which these laws are different, can it then be concluded that one of these theories might be confirmed and the other one refuted empirically? The answer is negative. Empirical observations provide us with information that has little relevance to this question. The answer is given by referring to the philosophical basic assumptions of science. First, it is a self-contradiction to postulate a unified theory that holds two different theories (in the sense that derivations of one theory are the opposite of those of another); second, it is no more than paraphrasing the negation of the "unity in variety" self-evident proposition of science.

Finally, in the light of the above, one might question the scientific significance of the empirical demonstration that $\mathrm{Rc}$ is learned instrumentally. In essence, such a demonstration will show that both procedures share some procedural elements in common, a result that may be of great importance for the development of a unified theory of learning. For other reasons, N. E. Miller and Dworkin (1974) discuss six different significant contributions of the area of visceral learning, without mentioning in that context the "one or two different laws of learning" problem. In other words, the empirical work has many important implications for other questions, but it cannot give an answer to the question of interest here.

\section{REFERENCES}

Bronowski, J. Science and human values. New York: Harper Colophon Books, 1965.

Cummins, R. Dispositions, states and causes. Analysis, 1974, 34, 194-204.

Kimble, G. A. Hilgard and Marquis' conditioning and learning (2nd ed.). New York: Appleton-Century-Crofts, 1961.

Kunn, T. S. The structure of scientific revolutions (2nd ed. enlarged). Chicago: University of Chicago Press, 1970.

MACKINTOSH, N. J. The psychology of animal learning. New York: Academic Press, 1974.

Miller, N. E. Learning of visceral and glandular responses. Science, 1969, 163, 434-445.

Miller, N. E., \& Dworkin, B. R. Visceral learning: Recent difficulties with curarized rats and significant problems for human research. In P. A. Obrist, A. H. Black, J. Brener, \& L. V. DiCara (Eds.), Cardiovascular psychophysiology: Current issues in response mechanisms, biofeedback and methodology. Chicago: Aldine Press, 1974.

Miller, S., \& Konorski, J. On a particular type of conditioned reflex. Proceedings of the Biological Society, 1928, 99, 1155-1157.

SKLAR, L. Types of inter-theoretic reduction. British Journal of Philosophy of Science, 1967, 18, 109-124.

SuPPE, F. The search for philosophic understanding of scientific theories. In F. Suppe (Ed.), The structure of scientific theories. Urbana: University of Illinois Press, 1974.

(Received October 24, 1978.) 